\title{
La dimensión axiológica de la Cultura de Paz
}

Erwin Silva ${ }^{1}$

Recibido: 4 de septiembre de 2013 / Aprobado: 15 de noviembre de 2013

\section{La Cultura de Paz, matriz de valores. El concepto de Cultura de Paz.}

$\mathrm{E}$ n el breve recorrido de la cultura de paz, encontramos momentos en los que puede considerarse el desarrollo histórico y normativo de esta nueva cultura de convivencia humana que hace posible la vida actual y futura de la sociedad global y la conservación del medio ambiente.

Importante es reconocer que ya en 1986 el jesuita Felipe Mac Gregor Rolino aplicaba este concepto que llenó de significación con su práctica en la solución del problema de la violencia en el

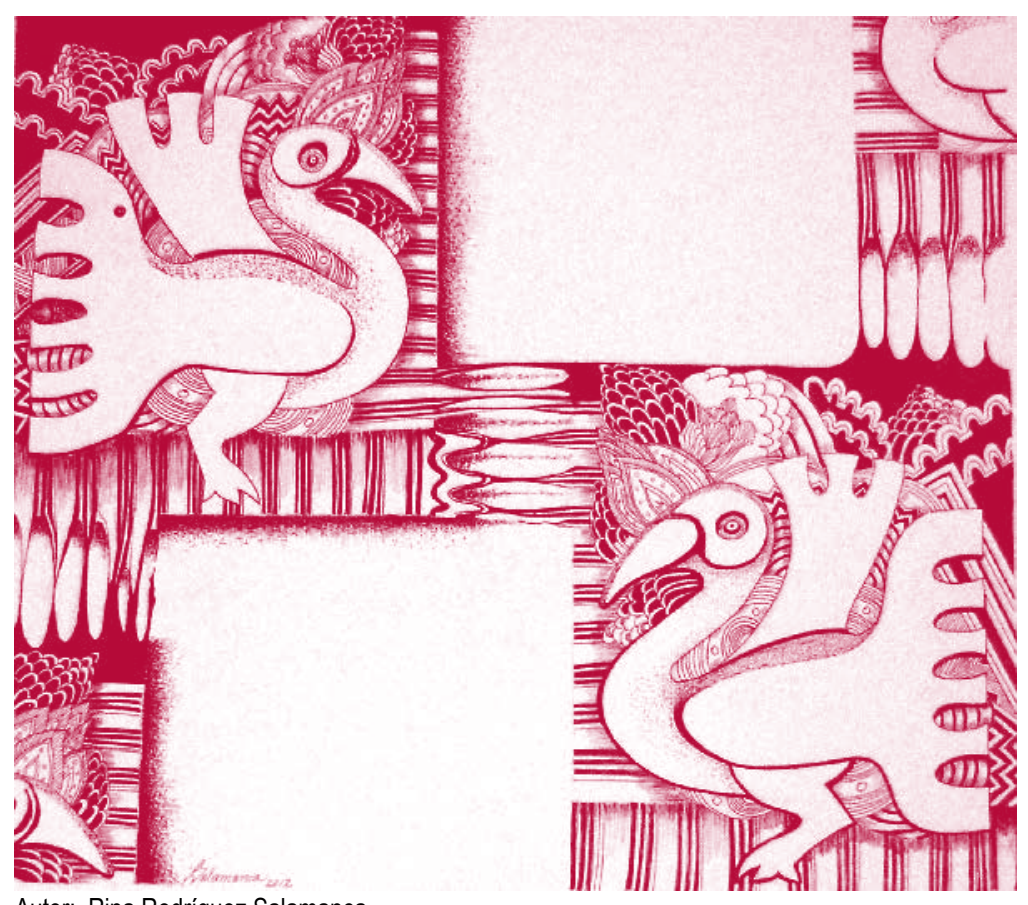

Autor: Rina Rodríguez Salamanca

1 Docente Investigador del Área de Educación para la Paz y los Derechos Humanos. Instituto Martin Luther King. Universidad Politécnica de Nicaragua (UPOLI). E-mail : erwinjotasil@gmail.com

\section{RESUMEN}

Este artículo es un breve análisis de los principios y valores de la Cultura de Paz presentes en los textos normativos, declaraciones y programas de acción de la Organización de las Naciones Unidas y la posibilidad de enseñarlos a las futuras generaciones. En lo fundamental se trata de mostrar los valores universales de la cultura de la paz constituidos en estos documentos que nos permiten ver esta dimensión valórica necesaria para establecer un nuevo paradigma global y pacifico de la convivencia humana.

Palabras clave: Cultura, Cultura de Paz, paz, axiología, educación, valores, organización de Naciones Unidas.

\section{ABSTRACT}

This article is a brief analysis of the principles and values of the Culture of Peace in the normative texts, statements and action programs of the United Nations Organization and the possibility to teach them to future generations. Fundamentally, it tries to demonstrate the universal values of the culture of peace constituted in these documents which allow us to see this dimension of values, necessary to establish a new global and peaceful paradigm of human coexistence.

Key words: Culture, Culture of Peace, peace, axiology, education, values, United Nations Organization. 
Perú. Entonces cultura de paz era todo el esfuerzo que debía hacerse para no emplear la violencia en la solución de los conflictos. La obra de él y de otros que le acompañaron en el proyecto de carácter educativo, se tituló "Cultura de Paz" ${ }^{2}$

Seguido con la Declaración de Yamousssukro sobre la paz en la mente de los hombres, Costa de Marfil de 1989, se adoptó el concepto de Cultura de Paz. Con una expresión en enunciados o aforismos que hallamos en los siguientes lemas en la Declaración de Yamousssukro:

"La paz es esencialmente el respeto de la vida. La paz es el bien más preciado de la humanidad. La paz no es solo el término de los conflictos armados. La paz es una adhesión profunda del ser humano a los principios de libertad, justicia, igualdad y solidaridad entre todos los seres humanos.

La paz es también una asociación armoniosa entre la humanidad y el medio ambiente.

Hoy en día, en vísperas del siglo XXI, la paz está a nuestro alcance."

En ese mismo año se emitió el Manifiesto de Sevilla de 1989 que plasma unas proposiciones sobre la violencia que permanecen irrebatibles como es la de sostener que la violencia no es genéticamente programada y concluyen con que la misma especie que ha inventado la guerra es capaz de inventar la paz. ${ }^{3}$

Otras declaraciones que constituyen norma son la Declaración y Programa de Acción sobre una Cultura de Paz. A/RES/ 53/243 (1999) y la Resolución de la Asamblea General de la ONU, denominada Década Internacional de una cultura de paz y no violencia para los niños del mundo, 2001-2010. (2001)

Y es aquí con estos fundamentos en los contenidos de estos trascendentales documentos que se despliega nuestro análisis.
Lo primero que queremos anotar es la definición del concepto de cultura de paz que extraemos de la Declaración y Programa de Acción sobre una Cultura de Paz (1999). Y se define así en el artículo 1:

"Una cultura de paz es un conjunto de valores, actitudes, tradiciones, comportamientos y estilos de vida basados en el respeto a la vida, el fin de la violencia y la promoción de la no violencia, por medio de la educación, el diálogo y la cooperación. (...)

Esta es una definición antropológica amplia y a simple vista se encuentra que la cultura de paz es un conjunto de valores que se tienen para la existencia y la construcción una cultura de vida no violenta, una cultura del diálogo o sea de la comunicación pacífica entre seres humanos y entre culturas, la cooperación que nos ha hecho humanos en la evolución social, y en lo fundamental, que se efectúa por medio de la educación. Esta es la dimensión axiológica de una Cultura de Paz puesto que se construye con valores y se admite la posibilidad de crear nuevos significados para los valores.

Marek Thee dijo hace algún tiempo que el concepto cultura de paz está altamente cargado de valores y la amalgama de la noción de cultura y la idea de paz con igual énfasis le confiere al concepto una particular importancia e intensidad. ${ }^{4}$ MarekThee propone una cultura de paz basada en los derechos humanos.

¿Cuáles son los valores de una Cultura de Paz? Indudablemente son los valores de la paz, el respeto a la vida, la libertad, la justicia, la solidaridad, la tolerancia, la igualdad entre hombres y mujeres. Si se efectúa una lectura detenida del texto clave de la Cultura de Paz citado ya, encontramos todos estos valores implícitos en las bases de la Cultura de la Paz.

El valor de la paz está realmente asociado al conjunto y forma, por decirlo así, una matriz de valores con nuevos significados que se cobran en el tiempo. La paz no es sólo

2 PNUD y Ministerio de Educación. Cultura de Paz3a .ed. Perú, 1989.

3 Silva, Erwin: Educación para una cultura de paz. Grupo Cívico. Ética y Transparencia, Nos-Otros, UPOLI, Managua, Nicaragua, 2010,154-157.

4 MarekThee. Towards a culture of peace based on Human Rights En: Culture of Peace and Democracy. UNESCO, Moscow, $1997,297$. 
ausencia de conflictos sino que es un proceso dinámico, positivo y participativo según dicha Declaración. La paz podemos decir es una actitud que vale siempre frente a los retos de la violencia.

La paz, volvemos a sostenerlo, no es lo opuesto a la guerra como quieren ver los teóricos del realismo político, sino que la paz es lo opuesto a la violencia. Ver la paz y la guerra en este juego binario de simples oposiciones, sería concebir una cierta visión simplista, determinista $\mathrm{y}$ alternante de guerra / paz en un círculo sin fin que no supera nunca la paz negativa y estaríamos siempre en los preparativos para la próxima confrontación bélica. Una de las bases epistemológicas de la Cultura de Paz no se encuentra en el sistema guerra/paz, porque esto sería un fatalismo sino en la paz positiva. Se dice que los realistas aceptan la realidad tal como está, la aceptan y se resignan, los que sostienen los mecanismos idealistas de la paz, quieren cambiar la realidad y emprenden la acción. Por la concepción de la paz positiva aplicada se va a la reducción o la eliminación de la violencia y la guerra no es más que la manifestación más alta y destructiva de la violencia. El concepto de paz positiva es una contribución de los estudios de paz y la investigación de Johan Galtung. ${ }^{5}$ Incluso, es posible denominar a un nuevo tipo de violencia que puede ser llamada violencia de la técnica que Ernst Jünger y Martin Heidegger habían visto como "la expresión conjunta de la racionalidad mundial y la voluntad de poder". ${ }^{6}$

Para el filósofo español Xabier Etxeberría, la paz positiva está ligada al desarrollo integral y los derechos humanos. $X$. Etxeberría concibe que la paz plena es la realización de todos los derechos humanos en su indivisibilidad, o sea de todos los tipos de derechos humanos que hasta hoy se han declarado en los textos normativos de la
Organización de las Naciones Unidas. ${ }^{7}$ Recordemos que una característica de los derechos humanos es que son indivisibles y no podemos dividirlos arbitrariamente para su cumplimiento o su vigencia y por lo tanto, se deben respetar todos los derechos humanos y no hay jerarquía entre ellos, sólo un orden en su aparición el tiempo.

También la paz como la violencia pueden verse en el origen y es clásico el lema de la Constitución de la UNESCO cuando dice que la guerra comienza en la mente de los hombres y es por eso que es ahí donde deben construirse los baluartes de la paz. De tal manera, que es dentro de los hombres y mujeres donde comienza la violencia, los hechos y palabras violentas son una manifestación de la división, el odio y la desconfianza que establece mi yo.

Mohandas Karamchad Gandhi, dice en su libro "Todos los hombres son hermanos", que primero está la intención violenta de dañar al adversario, o sea que los actos o dichos con que hago la violencia son una expresión de aquella violencia que está en mí. ${ }^{8}$ De igual manera Jiddhu Krishnamurti, dice que observemos la violencia en nosotros mismos y luego miremos la violencia externa. Para J. Krihsnamurti, somos seres humanos violentos. ${ }^{9}$ El conflicto no cesa porque el ego hace división entre el observador y lo observado y mi centro alberga el temor, el miedo, la codicia y la desesperación. Solo podemos cambiar el afuera cuando hemos hecho un cambio radical dentro de nosotros.

La no violencia es una base natural de la cultura de paz y de por sí, es la superación de la violencia por medio de la resistencia pacífica activa y por los métodos de resolución pacífica de conflictos, o sea, por medio del diálogo, la negociación, la conciliación, la mediación y el arbitraje.

5 Galtung, Johan. Peace by peaceful means, Peace and Conflict, development and Civilization. London, Sage Publications. 1996, 9.

6 Autores varios. La violencia y sus causas. UNESCO, París, Francia.1981, 41.

7 Etxeberría, Xabier. La concepción de paz positiva en: Hacia una cultura de paz. Colección Intertextos.No.5, Perú, 2009,40-45.

8 Gandhi, Mohandas. Todos los hombres son hermanos, Salamanca. España, 1988,146.

9 Krishnamurti, Jiddhu. Más allá de la violencia. EDHASA, Barcelona, 1991, 5 
En este arte de la no violencia destaca la eminencia de Mohandas K. Gandhi, quien contribuyó a la independencia de la India con acciones no violentas y valientes soportadas con el satyagraha, que es en esencia sostenerse con la fuerza de la verdad en una lucha sin violencia. Esto constituyó en ese tiempo una hazaña sin paralelo. Para Gandhi, ahimsa (noviolencia) y la verdad son lo mismo y ahimsa viene a ser la caridad perfecta.

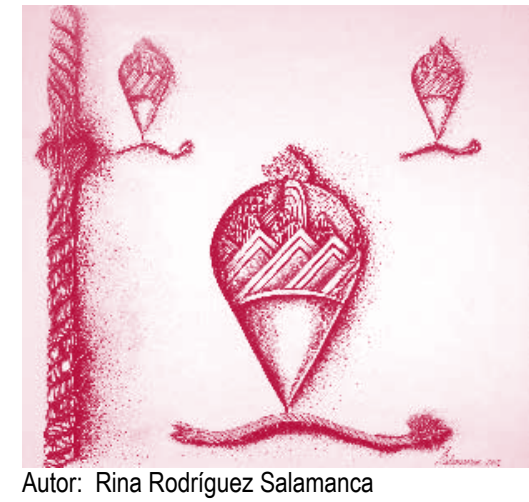

los imperios universales. La paz debe ser mi paz, en una relación que parte del yo y va hacia el Otro, en el deseo y la bondad donde el yo, a la vez, se mantiene y existe sin egoísmo."11

Varios son los exponentes de la paz y la no violencia en la historia reciente de la humanidad .Martin Luther King, en mi concepto es un ejemplo extraordinario que vence en su demanda de derechos humanos con marchas, protestas
Para Gandhi, la no violencia es un principio universal, es virtud personal y social, abstención de toda forma de explotación, valentía y verdad.

Otros filósofos como Raimon Panikkar, Enmanuel Lévinas y algunos grandes líderes de los movimientos sociales por los derechos humanos de la estatura de Martin Luther King Jr., hablan así de la paz.

Raimon Panikkar, un gran maestro de espiritualidad, nos enseñó que la paz es un don y nos recuerda con un trasfondo de las religiones fundamentales unos sutras de la paz que además de ser textos precisos, semejan los dichos de una antigua sabiduría tal como estos que dicen: (III) "La paz no se deja conquistar para uno mismo ni imponer a los demás. La paz se recibe, a la vez que se descubre, y se crea. Es un fruto (del Espíritu)."

O este otro que afirma: (IX) "Sólo el perdón, la reconciliación y el diálogo continuo conducen a la paz y rompen la ley del "karma". ${ }^{10}$

Enmanuel Lévinas nos muestra que la paz no es un encierro solipsista sino que va en dirección al Otro. De verdad que la paz interior es un punto de partida y la unidad de lo plural la definición de la paz. En su obra magna "Totalidad e Infinito" dice:

"La unidad de la pluralidad es la paz y no la coherencia de elementos que constituyen la totalidad. La paz no puede identificarse, pues, con el fin de los combates que acaban faltos de combatientes, por el fracaso de unos y la victoria de otros, es decir, con los cementerios o públicas, sermones, oraciones, libros y cartas y se logra con su legado aun después de su muerte el reconocimiento de aquellos derechos civiles para los negros en Estados Unidos de América. El doctor y reverendo bautista estadounidense, Martin Luther King Jr., dejó escritos los principios de la no violencia que él particularmente experimentó en la práctica del movimiento de los derechos civiles en los años sesenta del siglo pasado. En Martin Luther King encontramos que:

1. La no violencia es el modo de vida de la gente valiente.

2. La no violencia busca ganar amistad y comprensión.

3. La no violencia busca derrotar la injusticia no a la gente.

4. La no violencia sostiene que el sufrimiento voluntario puede educar y transformar.

5. La no violencia elige el amor y no el odio.

6. La no violencia cree que el universo está del lado de la justicia.

Estos seis puntos claves podemos hallarlos en el texto de King titulado "My pilgrimage to Nonviolence" publicado en 1958. ${ }^{12}$ Además legó los pasos prácticos para una campaña no violenta en su Carta desde la cárcel de Birmingham. Estas cuatro fases básicas son: "Primero, la reunión de los datos necesarios para determinar si existen las injusticias, luego la negociación; después la autopurificación, y por último, la acción directa."13

10 Panikkar, Raimon. Paz y desarme cultural. Editorial Sal Terrae. Bilbao, España, 1993, 36,43.

11 Lévinas, Enmanuel. Totalidad e Infinito. Ensayo sobre la exterioridad. Ediciones Sígueme, Salamanca, España, 1987,310.

12 Martin Luther King. My pilgrimage to nonviolence en: Strive to Freedom

13 Antología de Martin Luther King. Prólogo y selección de Pedro Guillén .Colección Pensamiento de América. Segunda Serie. Volumen 9, México D.F, $1968,7$. 
Pero nuestro recorrido sería incompleto si no viésemos la paz como un derecho humano que va alcanzando mejores definiciones en los congresos mundiales y en el seno de las Naciones Unidas. Sólo con las tres últimas Declaraciones sobre el derecho a la paz, Luarca, (2006); Barcelona, (2010) y la de Santiago de Compostela, (2010), nos bastaría para considerar la paz como un derecho humano en una progresiva instauración en la conciencia de los pueblos, la sociedad civil global y los investigadores y teóricos de la paz. De modo que al evocar la paz y toda su historia en las antiguas y nuevas culturas, nos hallamos en un momento en que la paz no es sólo un estado de paz mental sino que se extiende a la comunidad, los estados, los pueblos, las culturas y las religiones del mundo. Otra es la perspectiva de la paz como un derecho humano universal:

"Es precisamente en esta comprensiva y clarísima Declaración de Santiago de Compostela del año 2010, donde se plasma la definición de la paz como un valor universal y precondición del disfrute de los derechos humanos. Además de reconocer la concepción positiva de paz que desde el enfoque de Johan Galtung, no es sólo ausencia de guerra sino la eliminación o reducción de todo tipo de violencia, sea ésta directa, estructural o cultural. Aún más, pienso que una perspectiva es la vía de la concreción de una Convención del Derecho Humano a la Paz, para que las sociedades del mundo no se queden en el plano meramente declarativo sino que haya un instrumento vinculante de los Estados, porque éstos son también titulares del derecho humano a la paz. La paz debe ser considerada como un valor y como un derecho." ${ }^{14}$

En realidad cuando se examinan los nueve temas del concepto de cultura paz del Artículo1 de la Declaración y Programa de Acción sobre una Cultura de Paz del año 1999, puede decirse que nos encontramos ante un compromiso global o de todos los estados miembros de la ONU y otro es el horizonte que se abre para todos los seres humanos que aspiran a una civilización de la paz.

\section{Cultura de Paz implica:}

1 El respeto a la vida.

2 El respeto pleno de los principios de soberanía, integridad territorial en independencia de los Estados.

3 El respeto pleno y la promoción de todos los derechos humanos.

4 El arreglo pacífico de conflictos.

5 Protección del medio ambiente de las generaciones presentes y futuras.

6 El respeto y promoción del derecho al desarrollo.

7 El respeto y el fomento de la igualdad entre hombres y mujeres.

8 El respeto al derecho de libre expresión, opinión e información.

9 La adhesión a los principios de libertad, justicia, democracia, tolerancia, solidaridad, cooperación, pluralismo, diversidad cultural, diálogo y entendimiento y la paz.

Pero, ¿Cómo lograr el pleno desarrollo de una Cultura de Paz? La respuesta está en el artículo 2 de la Declaración y Programa de Acción, que dice:

"El progreso hacia el pleno desarrollo de una Cultura de Paz se logra por medio de valores, actitudes, comportamientos y estilos de vida propicios para el fomento de la paz entre las personas, los grupos y las naciones."

He aquí una de las formulaciones más breves y firmes de que en el núcleo del concepto de cultura de paz se asevera que la construcción de una cultura de paz tiene una dimensión axiológica. Es con los valores que vamos a edificar la paz y verla como una cultura que se vive entre personas e irradia a los grupos y las naciones enteras lo que hace ver una cultura de paz global. Es necesario afirmar que la Cultura de Paz es pragmática en el sentido que desemboca en la acción y la comunicación con los otros. Pero ante todo la Cultura de Paz es un trabajo con y por los valores.

14 Erwin Silva. El derecho humano a la paz. Elementos y perspectivas. En "Cultura de Paz" .57.Mayo-Agosto, 2011,23. 
La construcción de una Cultura de Paz también tiene una relación con las actitudes de empatía y no violencia, con los comportamientos éticos y políticos que tiendan al bien común y la solidaridad y con estilos de vida sencillos y que contribuyan a no tomar más de lo necesario de los recursos naturales y la explotación del mundo natural.

Otro documento importante en el desarrollo histórico normativo de la cultura de paz es el Manifiesto de Cultura de Paz del año 2000, que fue el Año Internacional de la Cultura de Paz, cuyos puntos esenciales transcribimos para glosar en cierto modo. Este documento fue suscrito por los Premios Nobel de Paz y afirma unos puntos claves que son:

- Respetar todas las vidas: Respetar la vida y la dignidad de cada persona sin discriminación ni prejuicios;

- Rechazar la violencia: Practicar la no violencia activa, rechazando la violencia en todas sus formas, física, sexual, sicológica, económica y social, en particular hacia los más débiles y vulnerables, como los niños y los adolescentes;

- Liberar la generosidad: Compartir mi tiempo y mis recursos materiales, cultivando la generosidad a fin de terminar con la exclusión, la injusticia y la opresión política y económica;

- Escuchar para comprenderse: Defender la libertad de expresión y la diversidad cultural, privilegiando siempre la escucha y el diálogo, sin ceder al fanatismo ni a la maledicencia y rechazo del prójimo;

- Preservar el planeta: Promover un consumo responsable y un modo de desarrollo que tenga en cuenta la importancia de todas las formas de vida y el equilibrio de los recursos naturales del planeta;

- Reinventar la solidaridad: Contribuir al desarrollo de mi comunidad, propiciando la plena participación de las mujeres y el respeto de los principios democráticos, con el fin de crear juntos nuevas formas de solidaridad.

Otro gran documento de alcance global importante de reconocer por la magnitud de la empresa y los propósitos nobles de su espíritu es la Declaración sobre el Decenio internacional de Cultura de Paz y No Violencia hacia los niños del mundo 2001 -2010.

El objetivo del Decenio fue en este caso, el de "fortalecer el movimiento mundial en pro de una cultura de paz y no violencia para los niños del mundo después de la celebración del Año Internacional de la Cultura de Paz en 2000."

Es admirable como en el concierto de las Naciones Unidas se concibe la importancia de los niños y niñas, puesto que éstos tienen capacidades para imaginar, trabajar, pensar y crear para vivir en un mundo nuevo sin aflicción ni violencia. Todos los niños del mundo tienen el derecho a crecer y vivir en paz y en un ambiente de felicidad.

\section{La educación para una Cultura de Paz como una educación en valores universales}

Las bases cognoscitivas, los valores universales, así como las destrezas, habilidades y competencias para educarnos en la cultura de paz, están inscritas en la Declaración y Programa de Acción sobre una Cultura de Paz del año 1999. Y, por supuesto, en todos los documentos sobre educación emitidos por la UNESCO en materia de educación para la paz, la democracia, la tolerancia y los derechos humanos.

Sin embargo, la primera noción que puede guiarnos en el trabajo de proponer esta nueva educación es la de Cultura y nos parece muy útil la que establece el filósofo Edgar Morin:

"La cultura está constituida por el conjunto de los saberes, saber -hacer, reglas, normas, interdicciones, creencias, valores, mitos que se transmiten de generación en generación, se reproducen en cada individuo, controlan la existencia de la sociedad y mantienen la complejidad psicológica y social." 15

Los términos cultura y paz se implican uno al otro, en razón de las normas y los valores, los comportamientos y los estilos de vida. Una cultura de paz se edifica por medio de la educación, como dice el propio texto de la

15 Morin, Edgar. Los siete saberes necesarios para la educación del futuro, Unesco, Francia, 1999,68. 
Declaración y Programa de Acción sobre una Cultura de Paz, Artículo 4:

"La educación a todos los niveles es uno de los medios fundamentales para edificar una cultura de paz. En ese contexto es de particular importancia la educación en la esfera de los derechos humanos."

Además de los gobiernos, la sociedad civil y los medios de difusión, son claves en el desarrollo de una cultura de paz.

La misma Declaración afirma en su Artículo 8, que:

"Desempeñan una función clave en la promoción de una cultura de paz los padres, los maestros, los políticos, los periodistas, los órganos y grupos religiosos, los intelectuales, quienes realizan actividades científicas, filosóficas, creativas y artísticas, los trabajadores sanitarios y de actividades humanitarias, los trabajadores sociales, quienes ejercen funciones directivas en diversos niveles, así como las organizaciones no gubernamentales."

Y dentro de los objetivos, estrategias y agentes de la presente Declaración, se sostiene que hay que:

"Velar por que los niños, desde la primera infancia, reciban instrucción sobre valores, actitudes y comportamientos y estilos de vida que les permitan resolver conflictos por medios pacíficos y en un espiritu de respeto por la dignidad humana y de tolerancia y no discriminación."

Las bases de una cultura de paz son realmente los temas que integran la educación para una cultura de paz y por cualquiera de estas vías se construye la paz y se han determinado de la siguiente forma:

1 El arreglo pacífico de conflictos.

2 El desarrollo sostenible

3 Derechos Humanos

4 Igualdad de género

5 Participación democrática

6 Comprensión, Tolerancia y solidaridad

7 Comunicación participativa y libre flujo de información

8 Paz internacional y seguridad

Para echar estas bases es indispensable concebir la educación como la herramienta fundamental en la tarea constante de la edificación de esta nueva cultura de la paz. Cada una de estas bases, temas o áreas de acción, implica un tipo de educación que conforma el conjunto de conocimientos, prácticas, valores, competencias y habilidades necesarias para la construcción de una cultura de paz global.

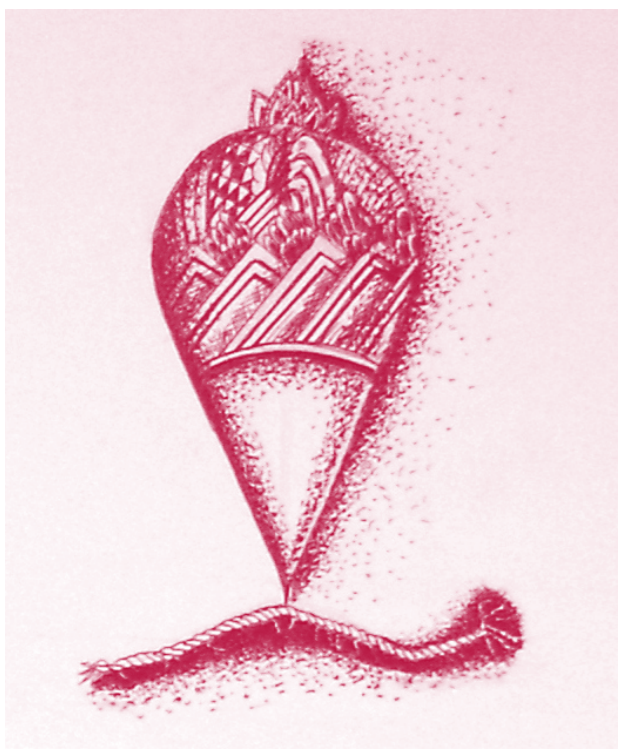

Autor: Rina Rodríguez Salamanca 